\title{
O LUGAR E O PAPEL DA PSICOLOGIA AMBIENTAL NO ESTUDO DAS QUESTÕES HUMANO-AMBIENTAIS, SEgundo Grupos de Pesquisa BRASILEIROS ${ }^{1,2}$
}

\author{
José Queiroz Pinheiro ${ }^{3}$ \\ Universidade Federal do Rio Grande do Norte
}

\begin{abstract}
Neste artigo, o autor responde às perguntas norteadoras baseado no banco de dados do Diretório Nacional de Grupos de Pesquisa do CNPq e no Banco de Teses da CAPES. Coloca-se inicialmente a partir de sua experiência profissional e como coordenador da REPALA, apresentando algumas características positivas da área e algumas das dificuldades enfrentadas. A partir da análise dos dados levantados discute, entre outros tópicos, a relação entre a psicologia e a Psicologia Ambiental, apontando a presença de várias psicologias ambientais. Apresenta sugestões para um desenvolvimento harmonioso e produtivo da área.
\end{abstract}

Descritores: Psicologia ambiental. Objeto. Grupos de pesquisa. Teses.

\begin{abstract}
Gostaria de começar minha apresentação pela analogia do elefante, que Robert Gifford apresentou anteriormente aqui, como uma metáfora para este nosso encontro. ${ }^{4}$ Imaginar que vamos conhecer todo o elefante é pretensão demais para um encontro desse porte. Isso iria demandar muito
\end{abstract}

1 O autor agradece ao CNPq, pelo apoio para a realização deste estudo, e a Dianne P. B. A. Sousa, bolsista de iniciação científica (Psicologia/UFRN), pela participação.

2 Transcrição: Kátia de Bonis.

3 Membro do corpo docente do programa de Pós-Graduação da Universidade Federal do Rio Grande do Norte e editor de Estudos de Psicologia. Endereço eletrônico: pinheiro@cchla.ufrn.br 
mais tempo mas, talvez, seja possível contribuirmos para que possamos identificar de que partes do elefante cada um de nós está tratando. E até ir um pouco além disso: não só conhecer a nossa própria parte, mas também conhecer as partes de que os colegas estão tratando. Isso é muito importante para podermos realizar uma cooperação entre grupos.

Meu referencial neste caso é a minha própria experiência como participante de um grupo de pesquisa na área e, mais recentemente, coordenador de uma lista de discussão na internet, chamada REPALA - Rede de Psicologia Ambiental Latino-Americana -, que tem servido para nos ensinar muito. Apresentarei, brevemente, apenas uma parte do estudo que havia planejado apresentar e, se houver pessoas interessadas, posteriormente poderei fornecer mais informações.

O primeiro ponto para o qual queria chamar a atenção é que quem trabalha em contato com turmas de graduação de Psicologia está acostumado a ver o aluno, aqui do Brasil, ter uma perspectiva muito positiva com relação à Psicologia Ambiental. Quando esses alunos ouvem falar pela primeira vez a respeito de Psicologia Ambiental, reagem dizendo algo como: "Ah! Que interessante?'. Parece que, para eles, faz sentido existir um ambiental na Psicologia.

Não é só o aluno de graduação que tem essa perspectiva. Em um $\mathrm{k}-$ vantamento realizado pela CAPES $^{5}$ (órgão do Ministério da Educação), entre pesquisadores da área de psicologia, procurourse conhecer as lacunas de formação pós-graduada. Os pesquisadores consultados apontaram a Psicologia Ambiental como a terceira lacuna mais importante, e algumas das justificativas apresentadas foram: "importância da área para a sociedade ou para determinada região", "inexistência ou insuficiência de programas", "importância da área por sua natureza interdisciplinar", "importância em outros países", "existe demanda de profissionais qualificados na área", e "é

4 Ver analogia do elefante no texto da comunicação apresentada por David Uzzell, às páginas 185-199.

5 CAPES: Coordenação de Aperfeiçoamento de Pessoal de Nível Superior 
área carente de integração com nossa realidade sociocultural" (BorgesAndrade, 2001, p. 166).

Nesse levantamento da CAPES, entretanto, não apareceram indicações de temas específicos em Psicologia Ambiental, mostrando o reconhecimento da necessidade de uma Psicologia Ambiental, mas na forma de algo não muito claro para os respondentes. Essa, aliás, é uma situação semelhante à encontrada no aluno de graduação e em outros interessados pela área. É justamente esse interesse meio vago que encontramos nas referências dos manuais, das obras que começaram a ser publicadas nos anos 1970, e após, a respeito de Psicologia Ambiental. Quando se observa a seção introdutória desses manuais, a maioria deles fala dos problemas ambientais, da superpopulação, do adensamento urbano, como justificativas para a existência dessa área. Desse modo, a Psicologia era confrontada com a necessidade de responder a essas demandas sociais, responder ao ambiental com o psicológico. Creio que parte dessa discussão que estamos tendo hoje aqui é justamente porque nós, psicólogos, e aqueles que estão atuando nessa área conosco, não temos sabido responder tão satisfatoriamente, como seria desejável, a essas demandas.

Temos, aqui no Brasil, trabalhado no sentido de nos aproximarmos uns dos outros. A área de Psicologia Ambiental esteve muito incipiente até mais ou menos o meio da década de 1990, e foi a partir daí, e principalmente nos últimos anos, que começou a surgir um conjunto de esforços para integrá-la, não só dentro da Psicologia, mas também integrando psicologia com outras áreas profissionais (Pinheiro, 2001). A propósito, essa integração com outras áreas do conhecimento decorre muito mais da iniciativa das outras áreas em se aproximar da Psicologia, como é o caso da Arquitetura e Urbanismo, do que da Psicologia estender a sua mão para fora.

Dentre as dificuldades encontradas pela Psicologia Ambiental no Brasil, a variedade de trajetórias de formação dos que atuam no setor acaba sendo um problema, pois a produção em Psicologia identificada sob o rótulo de Psicologia Ambiental ainda é pequena e bastante diversificada. Entre nós ainda se discute a definição de um campo específico e independente dentro 
da Psicologia, dedicado ao estudo das inter-relações humano-ambientais. Seja porque, na opinião de alguns, esses temas deveriam fazer parte da agenda de psicólogos de todas as sub-áreas que já existem ou, segundo outros, porque essa temática estaria melhor alojada em algum campo interdisciplinar de conhecimento, como "ambiente e comportamento", "percepção ambiental", "estudos do lugar", "ecologia social", etc.

Em decorrência dessa situação, realizei um levantamento, que tem enquadre semelhante aos trabalhos aqui apresentados por Esther Wiesenfeld e Maria Vittoria Giuliani, no sentido de buscar em um banco de dados - o Diretório Nacional de Grupos de Pesquisa, do $\mathrm{CNPq}^{6}$, órgão do Ministério da Ciência e Tecnologia - o que fazem os grupos de pesquisa de todas as áreas do Brasil em termos de Psicologia Ambiental. Isso é uma tarefa relativamente simples de ser realizada, e eu gostaria de aproveitar a ocasião para lembrar que o CNPq está preparando agora a quinta edição desse diretório, e seria muito oportuno fazermos uma atualização de nossas informações.

Os dados analisados se referem ao período do início de 1997 até junho de 2001 e são fruto de levantamento feito com base na expressão "psicologia ambiental", como busca exata. Vou aqui me referir apenas aos resultados principais. A área de conhecimento predominante da maioria dos grupos encontrados no levantamento é a Psicologia: $64 \%$ dos grupos que se autoidentificam como de psicologia ambiental são de psicologia. Observe-se que estas informações não dependem do $\mathrm{CNPq}$, ou de critérios externos. É o próprio pesquisador que define a área de conhecimento predominante em seu grupo e que está trabalhando, de alguma forma, em Psicologia Ambie ntal. A segunda classificada é a área de Arquitetura e Urbanismo, acrescida à de Planejamento Urbano. No total, essa segunda área compreende $18 \%$ dos grupos. Em seguida, encontram-se Educação, Sociologia e Engenharia e Recursos Florestais.

A incorporação da psicologia ambiental pelos grupos de pesquisa encontrados é bastante superficial. Apenas dois grupos usam a expressão "psi-

6 CNPq: Conselho Nacional de Desenvolvimento Científico e Tecnológico. 
cologia ambiental" na identificação do próprio grupo. Os demais a usam de diferentes formas: raramente na linha de pesquisa que adotam, ou na palavra chave dessa linha de pesquisa; na grande maioria dos casos utilizam a expressão "psicologia ambiental" nas produções realizadas, e sempre de uma maneira que identifica a Psicologia Ambiental muito mais como campo de estudo, do que como disciplina (Sommer, 2000). Assim, esse levantamento sugere que a Psicologia Ambiental não atende a necessidade de identificação de um grupo de pesquisa na realidade acadêmica brasileira, no sentido de os grupos não utilizarem a Psicologia Ambiental para se auto-identificarem, seja no título do grupo, seja na linha de pesquisa.

A presença da Psicologia Ambiental também pode ser investigada nas referências constantes das produções bibliográficas desses grupos, ou das orientações acadêmicas realizadas (iniciação científica, dissertações de mestrado ou teses de doutorado). A grande maioria da produção desses grupos é em resumos de trabalhos apresentados em congressos; há apenas alguns poucos artigos em periódicos científicos.

Aproveitando o contexto de orientação acadêmica, fiz um levantamento adicional no Banco de Teses da CAPES. Já estão disponíveis para o usuário de internet informações a respeito de teses de doutorado e dissertações de mestrado que tenham sido defendidas em instituições reconhecidas pelo Ministério de Educação. Fazendo o mesmo tipo de busca ("psicologia ambiental", como expressão exata em qualquer dos campos), foi possível encontrar quinze trabalhos: cinco de doutorado e dez de mestrado. Desses quinze trabalhos, apenas um tinha psicologia ambiental no seu título, os demais foram localizados na busca eletrônica porque usavam a expressão como palavra-chave ou, na grande maioria dos casos, por que a incluíam no resumo, em geral como fonte de informação (por exemplo, "esse estudo se baseia num conjunto de informações da área de Psicologia Ambiental").

Convém observar ainda que no Diretório Nacional de Grupos de Pesquisa do CNPq o usuário da internet pode também consultar os currículos dos pesquisadores. Assim, é possível constatar que a grande maioria dos pesquisadores que tem título de doutor e vínculo com psicologia ambiental 
possui doutorado bastante recente, o que pode ser visto como um dado promissor, porque se contrapõe à qualidade ainda incipiente da produção bibliográfica. Mais da metade dos doutores que figuram nesse levantamento concluíram o doutorado após 1995; apenas uns poucos estavam titulados antes disso. Eu chamo a atenção para esse dado, porque é importante considerarmos que leva alguns anos para um doutor se estabelecer institucionalmente, montar uma equipe de pesquisa, definir um programa de investigação, arregimentar alunos de pós-graduação, como sabem os colegas aqui presentes que trabalham com pós-graduação.

Um outro aspecto importante é que, no caso desses doutores com formação no país, não houve continuidade entre gerações acadêmicas. Os doutores que aparecem no levantamento porque indicam sua produção pessoal como sendo de Psicologia Ambiental foram formados por orientadores cuja produção é identificada como sendo de áreas como Psicologia Comunitária e Psicologia Social, entre outras. Ainda não temos entre nós uma segunda geração formada dentro do campo da Psicologia Ambiental. Portanto, o perfil que essa área vai assumir aqui no Brasil vai depender dos objetivos e esforços dos que tomarem para si a condução acadêmica, científica, mas também política dos fatos.

Os desdobramentos da Psicologia Ambiental em nosso país encontrarão um cenário mais complicado, até mesmo por causa das conhecidas rivalidades acadêmicas que, em minha opinião, ainda não afetaram diretamente a Psicologia Ambiental, por ela ainda ser "terra de ninguém", um território meio neutro justamente por não ter acesso a recursos, por não ter visibilidade na academia. O cenário tenderá a se tornar mais complicado também porque, como diz Wiesenfeld (2001), "uma vez consolidadas as formas alternativas de conhecer, investigar e construir conhecimento, a essa diversidade temática inicialmente detectada, somam-se modos não necessariamente complementares, mas inclusive opostos, de enfrentar os desafios a que nos propomos" (p. 38).

Alguns dos participantes daquela lista de discussão que mencionei antes, a REPALA, parecem estar cientes dessas dificuldades e dispostos a con- 
tinuar a bordo desse barco. Em uma das mensagens, uma das participantes dizia: "temos agora uma oportunidade ímpar para discutirmos questões sérias da área", e depois acrescentava: "e o nome que se dá a essa área, certamente, vai ainda trazer mais tensão e incertezas, tanto na geração de conhecimento como, principalmente, na prática profissional". "Essa discussão não é nova", ela dizia, "mas ainda está em pauta" (U. R. Santee, 21 de novembro de 2001).

Em função desse levantamento e de minhas experiências prévias na área, gostaria de encaminhar algumas reflexões relacionadas à discussão que estamos tendo neste encontro.

Gostaria de apontar, ainda que de modo possivelmente repetitivo para alguns dos presentes, que temos, na verdade, várias psicologias ambientais. Em primeiro lugar, temos pessoas que se classificam como de Psicologia Ambiental, mas nitidamente fazem um trabalho de Psicologia; que elegeram tópicos relacionados direta ou indiretamente às questões ambientais, sendo essa a grande parcela de trabalhos na área. É a Psicologia que se volta para as questões ambientais. Em um segundo tipo, há o que eu chamaria de "o estudo dos aspectos psicológicos presentes nas interações humanoambientais", ou seja, a ênfase é nas relações humano-ambientais, e o recorte, psicológico. No terceiro caso teríamos aquilo que aqui no Brasil passou a ser chamar de "o Ambiental", uma definição vaga não por acidente, uma caracterização bem ampla para poder arregimentar profissionais de diferentes formações (um exemplo deste caso poderia ser a recém criada ANPPAS, Associação Nacional de Pós-graduação e Pesquisa em Ambiente e Sociedade). $\mathrm{O}$ enquadre de uma pessoa nesses três tipos vai depender dos propósitos de cada um, de características pessoais, da formação teórica e profissional, dos recursos institucionais de que a pessoa dispõe, etc., não sendo incomum a atuação do indivíduo em mais de uma dessas situações. Eu me lembro, por exemplo, que, anos atrás, em um Congresso da SIP (Sociedade Interamericana de Psicologia), Gabriel Moser sugeria que nós, psicólogos ambientais, deveríamos participar de eventos que fossem eminentemente psicológicos e também de eventos necessariamente interdisciplinares. Vários de nós fazemos isso, participando, por exemplo, das reuniões da SIP e, ao mesmo tem- 
po, dos encontros da IAPS (International Association for PeopleEnvironment Studies) e/ou da EDRA (Environmental Design Research Association). Fazer parte de um grupo torna essa estratégia ainda mais fácil e produtiva, pois seus integrantes podem se envolver em diferentes entidades ou associações, com óbvios ganhos para os trabalhos do grupo.

Considerando principalmente a presença de alunos de graduação e de pós-graduação, gostaria também de chamar a atenção para a herança que a Psicologia Ambiental traz da própria Psicologia, como no caso do modelo médico, cujo posicionamento remediativo entra em choque direto com a postura preventiva característica da Psicologia Ambiental. Assim, em vez de estudar a preocupação ambiental, talvez devamos trabalhar com comprometimento ambiental, como fazem os psicólogos organizacionais e de outras áreas. Na verdade, estamos interessados no comprometimento das pessoas com o ambiente à sua volta, e não com a preocupação que elas têm com o ambiente. A Psicologia vive dizendo que, para se ter qualidade de vida, deve-se reduzir o estresse, evitar preocupação. Devemos, portanto, estudar e incentivar o comprometimento, e não a preocupação.

Um outro aspecto, relacionado à intervenção de Gary Evans hoje, e também com o que disse Aziz Ab'Saber ontem, refere-se à estratégia indutiva. Precisamos, por exemplo, localizar os bem-sucedidos em comprometimento ambiental e aprender com eles. Chamo de bem-sucedidos aquelas pessoas que, na contramão de todas as pressões sociais, econômicas, etc., ainda fazem reciclagem de lixo, coleta seletiva, organizam os condôminos do prédio, etc. Nós precisamos conhecer, aprender a ouvir essas pessoas, reconhecer nelas as características que queremos promover.

Realizamos ontem um (agradável) "passeio ambiental" pelos arredores da cidade de São Paulo e, logo após, uma avaliação do passeio. Entre as pessoas que avaliaram o passeio, somente uma mencionou as pessoas, os demais participantes mencionaram apenas aspectos do ambiente físico, como bem observou David Uzzell. Ele chamou nossa atenção para o fato de que os psicólogos não tinham falado de gente, tinha sido uma arquiteta quem falou de gente. É irônico mas, por outro lado, é positivo, porque sina- 
liza para a existência daquela ponte entre áreas que alguém que me antecedeu estava considerando necessária.

Um outro exemplo dessa ponte foi recentemente apresentado por Euclides Sánchez, durante o Segundo Encontro Latino-americano de Psicobgia Ambiental, no México. Ele contou que após atuarem interdisciplinarmente por vários anos no Conselho Nacional de Habitação da Venezuela, arquitetos e engenheiros haviam passado a considerar "o outro", fruto de um diálogo interprofissional arduamente construído. Tanto no caso do Conselho Nacional de Habitação, como no caso da arquiteta que viu gente, estamos diante de um movimento qualitativo para fora do nível das disciplinas; não é só uma passagem horizontal, uma ponte, mas algo supra-disciplinar.

Nós, psicólogos, quando olhamos para a equação proposta por Kurt Lewin - comportamento é função de pessoa em interação com ambiente, ou $\mathrm{C}=\mathrm{f}(\mathrm{P} \times \mathrm{A})$-, vemos o "P" maiúsculo, e o "a", lá atrás, pequenino. A pessoa é figura, e o ambiente fundo. Os arquitetos, geógrafos, planejadores urbanos, ao contrário, olham aquele parênteses da fórmula de uma outra perspectiva: vêm o "A" em primeiro plano, como figura, e o "p", de pessoa, lá longe, como fundo. $\mathrm{O}$ grande desafio que temos pela frente é conseguir enxergar "P x A" como uma unidade. Isso não é propriamente novidade. Roger Barker, em 1968, disse que a ciência que iria estudar o behavior setting talvez não fosse a Psicologia, tal como então estabelecida.

Para conseguir enxergar "P x A" como figura, precisamos descobrir o que vai ser fundo. Porque, ao aceitar os princípios da gestalt de figura e fundo, para que "P x A" seja figura, é preciso haver algo contrastando. Minha sugestão para esse "fundo" é a noção de sustentabilidade, a proporcionar um referencial para o binômio pessoa-ambiente. Essa noção, por mais vaga que seja, fornece um elemento, um apoio para o movimento pretendido, indo além do campo do que é puramente psicológico, passando pelos aspectos psicológicos das relações humano-ambientais, e chegando ao que chamei acima de "o ambienta".

Gostaria de concluir, chamando a atenção para ganhos que a noção de sustentabilidade traz para o nosso trabalho. Em primeiro lugar, ela não é 
domínio de nenhuma disciplina acadêmica, o que é uma grande vantagem. Se ela é imprecisa, por outro lado ela não é território de ninguém e pode servir de guarda-chuva, de objetivo comum, de ponto de convergência entre Psicologia Ambiental e outras áreas de conhecimento dedicadas ao estudo das relações pessoa-ambiente.

Via sustentabilidade, a Psicologia Ambiental poderia também se aproximar de outras áreas da própria Psicologia. Embutida na noção de sustentabilidade está a questão da solidariedade intra-geração, a questão da cooperação, o que faz uma ponte fantástica com a Psicologia Social. Quando consideramos a solidariedade entre gerações - nós e as gerações futuras temos uma ponte muito interessante com outros domínios psicológicos, no sentido de entender como funciona a perspectiva temporal para se alcançar a sustentabilidade.

Pinheiro, J. Q. (2005). The place and role of environmental psychology in the study of human-environmental issues, according to brazilian research groups. Psicologia USP, 16(1/2), 103-113.

\begin{abstract}
In this article, the author answers the guiding questions based on the database of Diretório Nacional de Grupos de Pesquisa do CNPq (research groups enrolled at $\mathrm{CNPq}-$ Brazilian Council of Scientific and Technological Development), and on the Collection of Theses of CAPES (Brazilian Ministry of Education's foundation for the qualification of higher education professors). He begins by commenting on his professional experience and by mentioning that he is the coordinator of REPALA (Latin-American Environmental Psychology Network), presenting some positive characteristics of the area and some difficulties that have been faced. Based on the data analysis he discusses, among other topics, the relation between Psychology and Environmental Psychology, pointing to the existence of many Environmental Psychologies. He presents suggestions for a harmonious and productive development of the area.
\end{abstract}

Index terms: Environmental psychology. Object. Research groups. Theses. 
Pinheiro, J. Q. (2005). Le lieu et le rôle de la psychologie de l'environnement dans l'ètude des questions humaines-de l'environnement, suivant des groupes de recherche brésiliens. Psicologia USP, 16(1/2), 103-113.

Résumé: Dans cet article, l'auteur répond aux questions dirigées, basé sur la banque de données du Directoire National de Groupes de Recherche du $\mathrm{CNPq}$ et sur la Banque de Thèses de la CAPES. Il s'affirme initialement conformément à son expérience professionnelle et en tant que coordinateur de la REPALA, manifestant quelques caractéristiques positives du domaine ainsi que quelques -unes unes des difficultés affrontées. À partir de l'analyse des données rassemblées, il discute, entre autres sujets, de la relation entre la Psychologie et la Psychologie de l'Environnement, signalant la présence de différentes Psychologies de l'Environnement. Il présente des suggestions pour un développement harmonieux et productif de ce domaine.

Mots-clés: Psychologie de l'environnement. Objet. Groupes de recherche. Thèses.

\section{Referências}

Barker, R. G. (1968). Ecological psychology. Stanford, CA: Stanford University Press.

Borges-Andrade, J. E. (2001). Seminário Nacional "Pós-graduação: enfrentando novos desafios - Psicologia”. Infocapes, 9(2/3), 166-174.

Pinheiro, J. Q. (2001). (Um pouco da) psicologia ambiental no Brasil: identidade, incertezas, perspectivas. In E. Tassara (Org.), Panoramas interdisciplinares para uma psicologia ambiental do urbano (pp. 11-26). São Paulo: EDUC / FAPESP.

Sommer, R. (2000). Discipline and field of research: A search for clarification. Journal of Environmental Psychology, 20, 1-4.

Wiesenfeld, E. (2001). Tendencias y perspectivas de desarrollo en Psicología Ambiental. In E. Tassara (Org.), Panoramas interdisciplinares para uma psicologia ambiental do urbano (pp. 27-49). São Paulo: EDUC / FAPESP.

Recebido em 5.04.2004

Revisto e encaminhado em 23.02.2005

Aceito em: 7.03.2005 